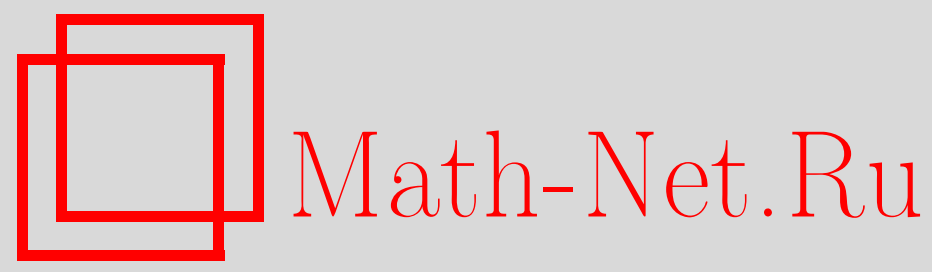

А. Н. Чупрунов, И. Фазекаш, Об обобщенной схеме размещения со случайным числом частиц, Дискрет. матем., 2012, том 24, выпуск 2, 149-153

DOI: https://doi.org/10.4213/dm1190

Использование Общероссийского математического портала Math-Net.Ru подразумевает, что вы прочитали и согласны с пользовательским соглашением http://www.mathnet.ru/rus/agreement

Параметры загрузки:

IP: 34.227 .88 .159

26 апреля 2023 г., 06:51:14 
УДК 519.2

\section{Об обобщенной схеме размещения со случайным числом частиц}

() 2012 г. А. Н. Чупрунов, И. Фазекаш

Показано, что обобщенная схема размещения с неполным комплектом частиц и другие аналоги обобщенной схемы размещения являются частными случаями обобщенной схемы размещения со случайным числом частиц.

Пусть $\xi_{0}, \xi_{1}, \xi_{2}, \ldots, \xi_{N}-$ независимые одинаково распределенные невырожденные случайные величины, принимающие значения в множестве неотрицательных целых чисел $\mathbf{N}_{0}, K$ - неотрицательная целочисленная случайная величина, не зависящая от семейства $\left\{\xi_{0}, \xi_{1}, \xi_{2}, \ldots, \xi_{N}\right\}$. Рассмотрим случайные величины $\eta_{1}, \ldots, \eta_{N}$ с совместным распределением

$$
\mathbf{P}\left\{\eta_{1}=k_{1}, \ldots, \eta_{N}=k_{N}\right\}=\mathbf{E}^{K} \mathbf{P}\left\{\xi_{1}=k_{1}, \ldots, \xi_{N}=k_{N} \mid \sum_{i=1}^{N} \xi_{i}=K\right\} .
$$

При этом предполагается, что

$$
\mathbf{P}\left(\xi_{1}+\xi_{2}+\ldots+\xi_{N}=K(\omega)\right)>0
$$

почти наверное.

Пусть

$$
n=k_{1}+k_{2}+\ldots+k_{N} .
$$

Допустим, что распределение случайной величины $K$ совпадает с распределением случайной величины

$$
S_{N}=\sum_{i=1}^{N} \xi_{i}
$$

Тогда, так как

$$
\mathbf{P}\left\{\eta_{1}=k_{1}, \ldots, \eta_{N}=k_{N}\right\}=\mathbf{P}\left\{\xi_{1}=k_{1}, \ldots, \xi_{N}=k_{N} \mid \sum_{i=1}^{N} \xi_{i}=k\right\}=0, \quad k \neq n,
$$

получаем, что

$$
\begin{aligned}
\mathbf{P}\left\{\eta_{1}=k_{1}, \ldots, \eta_{N}=k_{N}\right\} & =\mathbf{P}\left\{\xi_{1}=k_{1}, \ldots, \xi_{N}=k_{N} \mid \sum_{i=1}^{N} \xi_{i}=n\right\} \mathbf{P}\left\{\sum_{i=1}^{N} \xi_{i}=n\right\} \\
& =\mathbf{P}\left\{\xi_{1}=k_{1}, \ldots, \xi_{N}=k_{N}\right\} .
\end{aligned}
$$


Поэтому в этом случае совместное распределение случайных величин $\eta_{1}, \ldots, \eta_{N}$ совпадает с совместным распределением случайных величин $\xi_{1}, \ldots, \xi_{N}$ и схема независимых случайных величин является частным случаем схемы (1).

Обобщенная схема размещения является другим частным случаем схемы (1). Пусть случайная величина $K$ постоянна, т.е. $K=n$. Тогда мы получаем обобщенную схему размещения, введенную В. Ф. Колчиным в [1] (см. также монографии $[2,3])$, где случайные величины $\eta_{1}^{\prime}, \ldots, \eta_{N}^{\prime}$ имеют совместное распределение

$$
\mathbf{P}\left\{\eta_{1}=k_{1}, \ldots, \eta_{N}=k_{N}\right\}=\mathbf{P}\left\{\xi_{1}=k_{1}, \ldots, \xi_{N}=k_{N} \mid \sum_{i=1}^{N} \xi_{i}=n\right\} .
$$

Обобщенная схема размещения интерпретируется как схема размещения $n$ частиц по $N$ ячейкам. Поэтому схему (1) можно интерпретировать как обобщенную схему размещения случайного числа $K$ частиц по $N$ ячейкам.

В [4] был предложен аналог обобщенной схемы размещения - случайные величины $\eta_{1}^{\prime}, \ldots, \eta_{N}^{\prime}$, имеющие совместное распределение

$$
\mathbf{P}\left\{\eta_{1}=k_{1}, \ldots, \eta_{N}=k_{N}\right\}=\mathbf{P}\left\{\xi_{1}=k_{1}, \ldots, \xi_{N}=k_{N} \mid \sum_{i=1}^{N} \xi_{i} \in M\right\},
$$

где $M$ - такое подмножество $\mathbf{N}_{0}$, что $\mathbf{P}\left(\xi_{1}+\xi_{2}+\ldots+\xi_{N} \in M\right)>0$.

Рассмотрим случайные величины

$$
\mu_{M N}^{(r)}=\sum_{i=1}^{N} \mathbf{I}_{\left\{\eta_{i}=r\right\}}, \quad \eta_{(M N)}=\max _{1 \leqslant i \leqslant N} \eta_{i},
$$

где $\mathbf{I}_{\left\{\eta_{i}^{\prime}=r\right\}}$ - индикатор события, состоящего в том, что случайная величина $\eta_{i}^{\prime}$ равна $r$. Здесь $r-$ фиксированное целое неотрицательное число. Случайная величина $\mu_{M N}^{(r)}$ и случайная величина $\eta_{(M N)}$ интерпретируются как число ячеек в обобщенной схеме размещения частиц по $N$ ячейкам, содержащих $r$ частиц и максимальное число частиц в ячейке в предположении, что количество частиц принадлежит множеству $M$. Изучению случайных величин $\mu_{M N}^{(r)}$ и $\eta_{(M N)}$ в случае обобщенной схемы размещения посвящено большое количество работ (см. [2, 3] и их библиографию). При $M=\{0,1,2, \ldots n\}$ в [4] получены предельные теоремы для случайных величин $\mu_{M N}^{(r)}$ и случайных величин $\eta_{(M N)}$.

В [5] получены предельные теоремы для числа пустых ячеек в схеме размещения различимых частиц по ячейкам в случае неполных комплектов. Для модели (3) неполным комплектом являются случайные величины $\eta_{1}^{\prime}, \ldots, \eta_{l}^{\prime}, l<N$, имеющие совместное распределение

$$
\mathbf{P}\left\{\eta_{1}=k_{1}, \ldots, \eta_{l}=k_{l}\right\}=\mathbf{P}\left\{\xi_{1}=k_{1}, \ldots, \xi_{l}=k_{l} \mid \sum_{i=1}^{N} \xi_{i} \in M\right\} .
$$

Для схемы (4) справедливо представление (1).

Теорема 1. Пусть $l \leqslant N$, случайные величины $\eta_{1}^{\prime}, \ldots, \eta_{l}^{\prime}$ имеют совместное распределение (4). Тогда

$$
\mathbf{P}\left\{\eta_{1}=k_{1}, \ldots, \eta_{l}=k_{l}\right\}=\mathbf{E}^{K} \mathbf{P}\left\{\xi_{1}=k_{1}, \ldots, \xi_{l}=k_{l} \mid \sum_{i=1}^{l} \xi_{i}=K\right\},
$$


где случайная величина $K$ не зависит от семейства $\left\{\xi_{0}, \xi_{1}, \xi_{2}, \ldots, \xi_{N}\right\}$ и имеет распределение

$$
\begin{aligned}
\mathbf{P}\{K=k\} & =\mathbf{P}\left\{\sum_{i=1}^{l} \xi_{i}=k \mid \sum_{i=1}^{N} \xi_{i} \in M\right\} \\
& =\frac{\mathbf{P}\left\{\sum_{i=1}^{l} \xi_{i}=k\right\} \mathbf{P}\left\{\sum_{i=l+1}^{N} \xi_{i} \in M-k\right\}}{\mathbf{P}\left\{\sum_{i=1}^{N} \xi_{i} \in M\right\}} .
\end{aligned}
$$

Доказательство. Пусть $k_{1}+k_{2}+\ldots+k_{l}=n$. Тогда

$$
\mathbf{P}\left\{\xi_{1}=k_{1}, \ldots, \xi_{l}=k_{l} \mid \sum_{i=1}^{l} \xi_{i}=k, \sum_{i=1}^{N} \xi_{i} \in M\right\}=0, \quad k \neq n .
$$

Поэтому

$$
\begin{aligned}
& \mathbf{P}\left\{\eta_{1}=k_{1}, \ldots, \eta_{l}=k_{l}\right\} \\
& =\frac{\mathbf{P}\left\{\xi_{1}=k_{1}, \ldots, \xi_{l}=k_{l}, \sum_{i=1}^{l} \xi_{i}=n, \sum_{i=1}^{N} \xi_{i} \in M\right\}}{\mathbf{P}\left\{\sum_{i=1}^{N} \xi_{i} \in M\right\}} \\
& =\sum_{\{k: \mathbf{P}\{K=k\}>0\}} \frac{\mathbf{P}\left\{\xi_{1}=k_{1}, \ldots, \xi_{l}=k_{l}, \sum_{i=1}^{l} \xi_{i}=k, \sum_{i=1}^{N} \xi_{i} \in M\right\}}{\mathbf{P}\left\{\sum_{i=1}^{l} \xi_{i}=k\right\}} \\
& \times \frac{\mathbf{P}\left\{\sum_{i=1}^{l} \xi_{i}=k\right\}}{\mathbf{P}\left\{\sum_{i=1}^{N} \xi_{i} \in M\right\}} \\
& =\sum_{\{k: \mathbf{P}\{K=k\}>0\}} \frac{\mathbf{P}\left\{\xi_{1}=k_{1}, \ldots, \xi_{l}=k_{l}, \sum_{i=1}^{l} \xi_{i}=k, k+\sum_{i=l+1}^{N} \xi_{i} \in M\right\}}{\mathbf{P}\left\{\sum_{i=1}^{l} \xi_{i}=k\right\}} \\
& \times \frac{\mathbf{P}\left\{\sum_{i=1}^{l} \xi_{i}=k\right\}}{\mathbf{P}\left\{\sum_{i=1}^{N} \xi_{i} \in M\right\}} \\
& =\sum_{\{k: \mathbf{P}\{K=k\}>0\}} \frac{\mathbf{P}\left\{\xi_{1}=k_{1}, \ldots, \xi_{l}=k_{l}, \sum_{i=1}^{l} \xi_{i}=k\right\}}{\mathbf{P}\left\{\sum_{i=1}^{l} \xi_{i}=k\right\}} \\
& \times \frac{\mathbf{P}\left\{k+\sum_{i=l+1}^{N} \xi_{i} \in M\right\} \mathbf{P}\left\{\sum_{i=1}^{l} \xi_{i}=k\right\}}{\mathbf{P}\left\{\sum_{i=1}^{N} \xi_{i} \in M\right\}} \\
& =\sum_{\{k: \mathbf{P}\{K=k\}>0\}} \mathbf{P}\left\{\xi_{1}=k_{1}, \ldots, \xi_{l}=k_{l} \mid \sum_{i=1}^{l} \xi_{i}=k\right\} \mathbf{P}\{K=k\} \\
& =\mathbf{E}^{K} \mathbf{P}\left\{\xi_{1}=k_{1}, \ldots, \xi_{N}=k_{N} \mid \sum_{i=1}^{l} \xi_{i}=K\right\} \text {. }
\end{aligned}
$$

Равенство (5) доказано. Равенство (6) следует из (7). Теорема доказана. 
При $l=N$ из теоремы 1 вытекает следующее утверждение.

Следствие 1. Пусть случайные величины $\eta_{1}^{\prime}, \ldots, \eta_{l}^{\prime}$ имеют совместное распределение (4). Тогда

$$
\mathbf{P}\left\{\eta_{1}=k_{1}, \ldots, \eta_{N}=k_{N}\right\}=\mathbf{E}^{K} \mathbf{P}\left\{\xi_{1}=k_{1}, \ldots, \xi_{N}=k_{N} \mid \sum_{i=1}^{N} \xi_{i}=K\right\},
$$

где случайная величина $K$ не зависит от семейства $\left\{\xi_{0}, \xi_{1}, \xi_{2}, \ldots, \xi_{N}\right\}$ и имеет распределение

$$
\mathbf{P}\{K=k\}=\mathbf{P}\left\{\sum_{i=1}^{N} \xi_{i}=k \mid \sum_{i=1}^{N} \xi_{i} \in M\right\} .
$$

Полагая в теореме $1 M=\{n\}$, приходим к следующему утверждению.

Следствие 2. Пусть $l<N$ и случайные величины $\eta_{1}^{\prime}, \ldots, \eta_{N}^{\prime}$ являются обобщенной схемой размещения $n$ частии по $N$ ячейкам. Тогда

$$
\mathbf{P}\left\{\eta_{1}=k_{1}, \ldots, \eta_{l}=k_{l}\right\}=\mathbf{E}^{K} \mathbf{P}\left\{\xi_{1}=k_{1}, \ldots, \xi_{l}=k_{l} \mid \sum_{i=1}^{l} \xi_{i}=K\right\},
$$

где случайная величина $K$ не зависит от семейства $\left\{\xi_{0}, \xi_{1}, \xi_{2}, \ldots, \xi_{N}\right\}$ и имеет распределение

$$
\begin{aligned}
\mathbf{P}\{K=k\} & =\mathbf{P}\left\{\sum_{i=1}^{l} \xi_{i}=k \mid \sum_{i=1}^{N} \xi_{i}=n\right\} \\
& =\frac{\mathbf{P}\left\{\sum_{i=1}^{l} \xi_{i}=k\right\} \mathbf{P}\left\{\sum_{i=l+1}^{N} \xi_{i}=n-k\right\}}{\mathbf{P}\left\{\sum_{i=1}^{N} \xi_{i}=n\right\}} .
\end{aligned}
$$

Пример 1. Схема размещения $n$ различимых частиц по $N$ ячейкам является обобщенной схемой размещения $n$ частиц по ячейкам $N$, с пуассоновскими случайными величинами $\xi_{i}$, имеющими параметр $\lambda$, [1]. Поэтому случайные величины $\sum_{i=1}^{l} \xi_{i}, \sum_{i=l+1}^{N} \xi_{i}$ и $\sum_{i=1}^{N} \xi_{i}$ - пуассоновские случайные величины с параметрами, $l \lambda,(N-l) \lambda, N \lambda$, соответственно. Следовательно, из (9) получаем

$$
\mathbf{P}\{K=k\}=\left(\begin{array}{l}
n \\
k
\end{array}\right)\left(\frac{l}{N}\right)^{k}\left(1-\frac{l}{N}\right)^{n-k}, \quad 0 \leqslant k \leqslant n .
$$

Таким образом, случайная величина $K-$ биномиальная случайная величина с параметрами $p=l / N$ и $n$. Случайную величину $K$ можно интерпретировать следующим образом. Пусть $I_{i}-$ индикатор события, состоящего в том, что $i$-я частица оказалась в одной из первых $l$ ячеек и индикаторы $I_{i}, 1 \leqslant i \leqslant n$, независимы. Тогда $\mathbf{P}\left\{I_{i}=1\right\}=l / N$ и число частиц, которые оказались среди первых $l$ ячеек - случайная величина $K=\sum_{i=1}^{n} I_{i}$.

Пример 2. Рассмотрим обобщенную схему размещения $n$ частиц по $N$ ячейкам, где случайные величины $\xi_{i}$ есть биномиальные случайные величины с параметрами $(m, p)$, где $m \in \mathbf{N}, 0<p<1$ (при $m=1, \xi_{i}$ - индикаторы). Тогда $\sum_{i=1}^{l} \xi_{i}, \sum_{i=l+1}^{N} \xi_{i}$ и $\sum_{i=1}^{N} \xi_{i}$ 
есть биномиальные случайные величины с параметрами $(m l, p),(m(N-l), p),(m N, p)$, соответственно. Следовательно, из (9) получаем

$$
\mathbf{P}\{K=k\}=\frac{\left(\begin{array}{c}
m l \\
k
\end{array}\right)\left(\begin{array}{c}
m(N-l) \\
n-k
\end{array}\right)}{\left(\begin{array}{c}
m N \\
n
\end{array}\right)}, \quad 0 \leqslant k \leqslant n .
$$

Таким образом, $K$ - случайная величина, имеющая гипергеометрическое распределение с параметрами $m N, m l$. Случайную величину $K$ можно интерпретировать следующим образом. В урне имеются $m l$ белых и $m(N-l)$ черных шаров. Мы вынимаем из урны $n$ шаров, белые среди этих шаров распределяем по первым $l$ ячейкам, а черные - по последним $N-l$ ячейкам. Тогда случайная величина $K-$ количество белых шаров в этой урновой модели.

Пример 3. Рассмотрим схему размещения $n$ частиц по $N$ ячейкам, причем все комбинации частиц равновероятны (схема размещения неразличимых частиц) и введем дополнительное условие, что в каждой ячейке содержится не менее $r$ частиц. Это соответствует случаю (см. с. 30 в [1])

$$
\mathbf{P}\left\{\xi_{0}=k\right\}=p^{k-r}(1-p), \quad k=r, r+1, \ldots, \quad 0<p<1 .
$$

Поэтому, используя в (9) последнюю формулу на с. 30 в [1], получаем

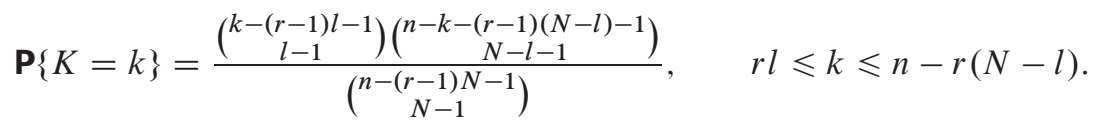

\section{Список литературы}

1. Колчин В. Ф., Один класс предельных теорем для условных распределений. Литовский матем. сборник (1968) 8, №1, 53-63.

2. Колчин В. Ф., Случайные графы. Физматлит, Москва, 2000.

3. Тимашёв А. Н., Асимптотические разложения в вероятностной комбинаторике. ТВП, Москва, 2011.

4. Чупрунов А. Н., Фазекаш И., Аналог обобщенной схемы размещения. Предельные теоремы для числа ячеек заданного объема. Дискретная математика (2012) 24, №1, 140-158.

5. Хакимуллин Е. Р., Энатская Н. Ю., Предельные теоремы для числа пустых ячеек. Дискретная математика (1997) 9, №2, 120-130.

Статья поступила 18.05.2011. 